\title{
Infertility and Hypergonadotropic Hypogonadism as First Evidence of Hereditary Apolipoprotein A-I Amyloidosis
}

\author{
Tiziano Scalvini, Paola Rossana Martini,* Laura Obici, Regina Tardanico, Luciano Biasi, \\ Gina Gregorini, Francesco Scolari and Giampaolo Merlini \\ From the Departments of Endocrinology and Andrology (TS, PRM) and Pathology (RT) and Divisions of Infectious Diseases (LB) and \\ Nephrology (GG, FS), Spedali Civili, Brescia and Amyloid Centre, Biotechnology Research Laboratories, Fondazione IRCCS Policlinico \\ San Matteo (LO, GM), Pavia, Italy
}

\begin{abstract}
Purpose: We report that primary infertility and hypergonadotropic hypogonadism in young patients may be caused by testicular amyloidosis and it is associated with the presence of a mutation in the apoA-I gene, resulting in the replacement of proline for leucine at residue 75 of the protein.

Materials and Methods: Ten patients presenting with infertility, gynecomastia, decreased libido, erectile dysfunction or a family history of amyloidosis underwent clinical evaluation, hormone assays, semen analysis, ultrasonographic investigation of the testicles, testicular biopsy and DNA sequencing of the apoA-I gene.

Results: All patients showed azoospermia and 9 had increased testicular volume. Massive amyloid deposition was observed in all testicular biopsies and the apoA-I mutation of replacement of proline for leucine at residue 75 of the protein was noted. Five patients showed hypergonadotropic hypogonadism and 5 had normal testosterone values with high gonadotropin levels. Conclusions: Nonobstructive azoospermia and macro-orchidism with or without hypogonadism may be caused by hereditary apoA-I amyloidosis in young patients. Testicular amyloidosis can be the first manifestation of this systemic disease. Specific staining for amyloid deposits and genetic analysis of apoA-I mutations are recommended in young, infertile patients with macro-orchidism. Finally, surveillance in asymptomatic mutation carriers is suggested to evaluate the opportunity to implement sperm retrieval and start androgen replacement therapy when necessary.
\end{abstract}

Key Words: testis; apolipoprotein A-I; infertility, male; amyloidosis; hypogonadism

$\mathrm{S}$ ystemic amyloidosis consists of a group of diseases characterized by the progressive extracellular deposition of soluble plasma proteins as insoluble fibrils, disrupting tissue integrity, which leads to organ dysfunction. ${ }^{1}$ Of the diseases $\mathrm{AL}$ and $\mathrm{AA}$ are the most frequent. Acquired AL is generated by the deposition of Ig light-chain fragments produced by clonal plasma cells, AA is a longterm complication of several chronic inflammatory conditions and some systemic types of amyloidosis are inherited and present as autosomal dominant diseases. In hereditary amyloidosis amyloid fibrils derive from a mutated form of 1 of certain proteins, including transthyretin, lysozyme, apoA-I, apolipoprotein A-II, fibrinogen $\mathrm{A} \alpha$ chain, cystatin $\mathrm{C}$ and gelsolin.

Clinically systemic amyloidosis is characterized by renal, heart, liver and peripheral nerve involvement that is isolated or variably associated, leading to progressive organ failure and ultimately to death. Testicular amyloid localization, resulting in azoospermia and hypogonadism, is rarely observed in the context of $\mathrm{AL},{ }^{2}$ while it is more frequently described in AA, especially in patients with familial Mediterranean fever. ${ }^{3,4}$ Testicular amyloidosis as a first sign of

Submitted for publication September 14, 2006.

Study received Institutional Review Board approval.

* Correspondence: Department of Endocrinology and Andrology, University of Brescia, Spedali Civili of Brescia, p. le Spedali Civili 1, 25123 Brescia, Italy (telephone: +39-0303995251; FAX: +39-0303388147; e-mail: prmartini@libero.it). systemic amyloidosis has been occasionally reported, manifesting as hypogonadism, sterility and macro-orchidism. ${ }^{5-7}$ However, in most of these cases it was never formulated as a diagnosis based on the biochemical nature of the amyloid deposits. Schrepferman et al described secondary infertility due to testicular amyloid deposition as the only clinical finding in a patient heterozygous for an apoA-I variant. ${ }^{8}$ Similarly testicular biopsy showed amyloid deposits several years before the occurrence of severe amyloid cardiomyopathy in a patient carrying the leucine 174 serine mutation of apoA-I. ${ }^{9}$

ApoA-I, the major component of high density lipoproteins, can cause systemic amyloidosis at the presence of specific amino acid replacements. A total of 12 amyloidogenic apoA-I variants have been identified to date. ${ }^{10}$ The natural history of the disease consists of progressive liver and kidney involvement with restrictive cardiomyopathy observed in some variants. We recently referred to an amyloidogenic variant of apoA-I associated with a predominantly sporadic presentation, in which the leucine residue at position 75 is replaced by proline (Leu75Pro). ${ }^{11}$ In the first 2 series of patients in whom the diagnosis was confirmed by genetic and immunohistochemical analyses surgically treated Gyn was reported in a few, ${ }^{11,12}$ suggesting testicular localization of the disease. However, no additional studies were performed in these subjects.

To our knowledge we report for the first time a detailed characterization of apoA-I testicular amyloidosis in a novel 
series of patients. Furthermore, this proves that the early deposition of amyloid in the testes leading to infertility is a peculiar feature of this form of the disease.

\section{MATERIALS AND METHODS}

Of 1,400 infertile men examined in a 13-year period at the Department of Endocrinology at University of Brescia 10 were found to have testicular amyloidosis due to the hereditary Leu75Pro mutation of Apo A-I. In all patients the testicle was the first or single organ involved. Mean patient age \pm SD was $35.7 \pm 6.3$ years (range 29 to 45 ) at diagnosis and all were included in the study. Patients were examined due to rLib, ED, gynecomastia, infertility or a family history of amyloidosis that was isolated or variably associated (see table).

All patients underwent testicular biopsy after collecting the personal and family medical history as well as physical examination of the genitals and breast, ultrasonographic investigation of the abdomen and testicles, and semen analysis. Blood samples for hormonal assays, hepatic and renal function parameters, and DNA sequencing of the apoA-I gene were also taken.

For the medical history particular attention was given to libido, erectile function, past urological pathologies, infertility, the number of children and patient age at conception. Assessment of the hormonal profile was performed at 9:00 a.m. with the patient fasting. The parameters measured at a centralized laboratory were LH, FSH, SHBG, T and calculated fT. Vermeulen's formula was used to calculate fT according to published criteria. ${ }^{13}$

At ultrasonographic investigation testicular volume was calculated with the Taskinen and Schiff formula, $0.71 \times$ length $\times$ width $\times$ depth. ${ }^{14}$ Echographic examinations were performed by the same physician using a 7 to $12 \mathrm{MHz}$ linear multifrequencing Sequoia Siemens ${ }^{\mathrm{TM}}$ instrument. In the literature a testicular volume of between 15 and $25 \mathrm{ml}$ is considered normal..$^{15}$ All data are presented as the mean \pm SD.

Semen analysis was performed by the same specialist according to the WHO manual definition. Histological evaluation was done by the same physician according to standard techniques. The search for amyloid deposits was done by Congo red staining, followed by microscopic examination under polarized light. Immunohistochemical characterization of amyloid deposits was made on paraffin embedded sections using a panel of antibodies against $\kappa$ and $\lambda$ chains, serum amyloid-A, fibrinogen and apoA-I (Dako, Milan, Italy). This last reaction was visualized with the alkaline phosphatase RED method.

All patients provided written informed consent and the study was approved by the Institutional Review Board. Genetic testing was performed on DNA extracted from peripheral blood using standard procedures. Screening for apoA-I mutations was done by direct sequencing of polymerase chain reaction amplified fragments, as previously described. ${ }^{11}$

\section{RESULTS}

The table lists patient initial clinical characteristics. Previous testicular pathologies were present in 3 cases, including varicocele, orchio-epididymitis and funicular torsion, respectively. Two patients had a family history of amyloidosis. Two

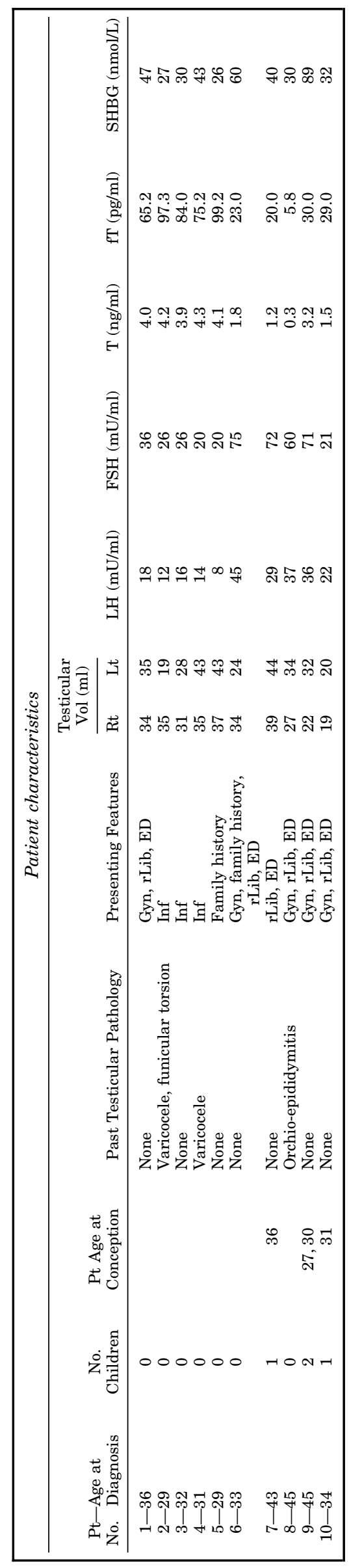


patients had fathered a child before diagnosis and 1 had fathered 2 children at the ages indicated (see table).

At physical examination 1 or 2 testicles appeared smooth, markedly enlarged and toughened in patients 1 to 9 . These findings were confirmed by a homogeneous solid appearance on ultrasound with a mean volume of $31.4 \pm 6.7 \mathrm{ml}$ (range 19 to 39 ) for the right testicle and $32.0 \pm 9.3 \mathrm{ml}$ (range 19 to 44 ) for the left testicle. No additional pathological findings were revealed by abdominal ultrasound screening.

All patients were completely azoospermic. Patient 2 underwent previous semen analyses due to infertility, which revealed normospermia $\left(32 \times 10^{6}\right.$ spermatozoa per $\mathrm{ml}$ ) 23 months before the diagnosis of amyloidosis and severe oligospermia $\left(60 \times 10^{3}\right.$ spermatozoa per $\left.\mathrm{ml}\right) 6$ months previously.

Hepatic and renal function was normal in all patients. Hypergonadotropic hypogonadism was present in patients 6 to 10 with low androgen levels, including $\mathrm{T} 1.6 \pm 1.1 \mathrm{ng} / \mathrm{ml}$ (normal at our laboratory 3.5 to 9 ) and fT $21.6 \pm 9.7 \mathrm{pg} / \mathrm{ml}$ (normal 75 to 124), and high gonadotropin values, including LH $33.8 \pm 8.7 \mathrm{mU} / \mathrm{ml}$ (normal 0.5 to 6 ) and FSH $59.7 \pm 22.4$ $\mathrm{mU} / \mathrm{ml}$ (normal 0.5 to 6 ). In contrast, patients 1 to 5 showed normal androgen values with $\mathrm{T} 4.1 \pm 0.2 \mathrm{ng} / \mathrm{ml}$ and fT $84.2 \pm 14.5 \mathrm{pg} / \mathrm{ml}$ but increased levels of $\mathrm{LH}$ and $\mathrm{FSH}$ at $13.7 \pm 3.9$ and $25.6 \pm 6.5 \mathrm{mU} / \mathrm{ml}$, respectively. All except patient 9 had SHBG concentrations within the normal range (13 to $71 \mathrm{nMol} / \mathrm{l})$.

Histological analysis of testicular biopsies showed abundant deposits of amorphous eosinophilic material, strongly suggestive of amyloid (part $A$ of figure). Congo red staining confirmed the presence of pathognomonic apple-green birefringence under polarized light. Amyloid deposits were found around the basement membrane of the seminiferous tubules, narrowing or obstructing the lumen in any case. Patients 3 to 10 had a germinal epithelium that was completely replaced by amyloid without Sertoli's cells. Patients 1 and 2 presented with mixed tissue morphology, showing areas with complete replacement of the germinal epithelium scattered with tubules with only Sertoli's cells as well as areas at all stages of spermatogenesis.

Amyloid deposits were also found in the interstitium, in the walls of arteries, capillaries and veins. They increased wall thickness with a decreased lumen in all patients, leading to complete obstruction in some men. No Leydig cells were seen in the biopsies of patients 6 to 8, who had hypo-

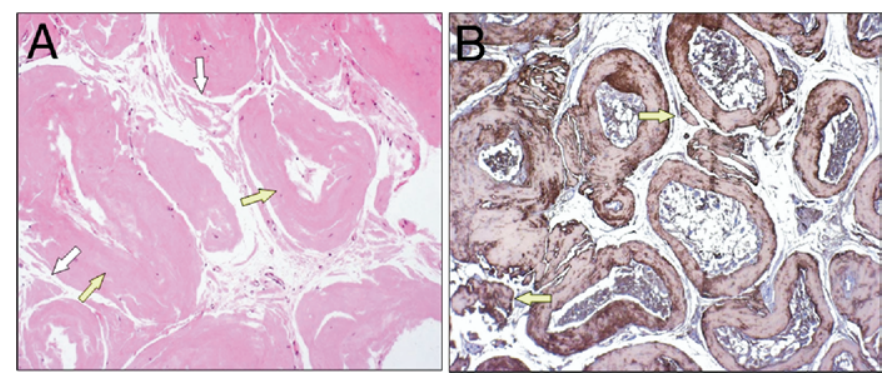

$A$, testicular biopsy reveals massive peritubular deposition of amorphous material with complete loss of germinal epithelium (yellow arrows). Interstitial amyloid deposits are visible focally (white arrows). $\mathrm{H} \& \mathrm{E}$, reduced from $\times 25 . B$, immunohistochemical analysis of testicular biopsy by anti-Apo A-I antibody shows intense immunostaining of peritubular and interstitial amyloid deposits, in particular focal interstitial deposits (yellow arrows). Reduced from $\times 25$. gonadism. Patient 9 with hypogonadism and patients 2 and 4 with normal testosterone levels showed only a few Leydig cells without precise tissue organization. Finally, patients 1 , 3 and 5 with normal testosterone and patient 10 with hypogonadism had few aggregates of Leydig cells, which retained normal tissue architecture. At immunohistochemical analysis amyloid deposits were intensively and specifically immunoreactive with anti-apoA-I antibody but not with the other antibodies tested (part $B$ of figure).

Genetic analysis performed through direct DNA sequencing showed that all 10 patients were heterozygous for a thymine to cytosine transition at nucleotide 1772 of the apoA-I gene, resulting in a leucine to proline amino acid replacement at residue 75 of the mature protein. No patients had different apoA-I variants, a normal apoA-I genotype or other sequence variations.

\section{DISCUSSION}

The current study demonstrates that testicular failure is a typical finding in hereditary apoA-I amyloidosis, especially in association with the Leu75Pro variant of this protein. Testicular involvement can be the first or the single manifestation of the disease and it evolves with time.

To our knowledge this is the first series of patients with apoA-I amyloidosis in which the clinical and pathological basis of testicular amyloid involvement was characterized in detail. These data strengthen and expand previous descriptions of single patients in whom testicular failure was reported in the medical history several years before a diagnosis of cardiac ${ }^{9}$ or visceral ${ }^{11,12}$ apoA-I amyloidosis was made.

The Leu75Pro mutation of the apoA-I gene leads to asymptomatic, long-standing, mild to moderate renal failure, and increased alkaline phosphatase and $\gamma$-glutamyltransferase levels. ${ }^{11,12}$ Compared to other variants this is associated with a mild phenotype due to the slower progression of kidney and liver deposits. The lack of a family history, advanced patient age at renal or hepatic biopsy and the limited evidence of disease related mortality strengthen this view. The current study proves that this disease occurs earlier than expected and it may be responsible for the dramatic impairment of fertility from the third decade of life. ApoA-I amyloidosis should be suspected in patients with azoospermia, a normal or decreased testosterone level and normal or high testicular volume even in the absence of an informative family history.

The frequency of this rare genetic condition still remains to be defined. A possible founder effect likely justifies the high incidence in our population. However, the same variant was reported in a patient with a different ethnic background, suggesting more widespread distribution. ${ }^{16}$

Our study shows the characterization of the pathological basis for testicular apoA-I amyloidosis and the possible molecular mechanisms of this disease. Biopsies revealed a germinal epithelium that was completely replaced by amyloid fibrils or Sertoli's cells only. Amyloid deposits are also present in the vessel walls, leading to narrowing or obstruction, and in the interstitium with partial or complete Leydig cell substitution. In patients 1 and 2 there coexisted areas of complete germinal epithelium replacement, areas with Sertoli's cells only and areas at all stages of spermatogenesis. Repeat semen analyses in patient 2 during the 23 months before diagnosis revealed rapid evolution from normosper- 
mia to oligospermia and azoospermia. The progressive impairment of spermatogenic function is due to the gradual deposition of amyloid, which causes parenchymal replacement, and it seems to reflect the mixed histological profile. Our findings agree with previous results, reflecting similar observations in $\mathrm{AA}$ and $\mathrm{AL}{ }^{4,17}$

To date different hypotheses have been formulated on the mechanisms that cause the impairment of spermatogenic function by amyloid. A hypothesis is that azoospermia can be due to the obliteration of intratesticular canaliculi or to the disruption of sperm production through a direct effect on the seminiferous tubules by amyloid deposits. ${ }^{3,4}$ Özdemir et al proposed that the narrowing and obstruction of vessels may cause long-term hypoxia, leading to disruption of the germinal epithelium with spermatogenic arrest. ${ }^{17}$ Vascular compression was proposed to be caused by amyloid deposition in the vessel tunica propria or by interstitial fluid from damaged and more permeable vessels.

In all patients testicular biopsies showed increased vessel wall thickness and partial or complete replacement of the germinal epithelium by amyloid deposits, indicating that tubular and vascular obstructions are probably the end stages of the same process. Amyloid fibrils were also found in the interstitium, causing partial or complete Leydig cell replacement. This explains the low level of T and fT, and the high level of gonadotropins in patients 6 to 10 . In patients 1 to 5 , in whom Leydig cells appeared less disrupted, high FSH and LH values were able to maintain normal testosterone levels, indicating early and subclinical gonadic dysfunction. Histological findings also support clinical and hormonal data, suggesting that testicular failure results from progressive parenchymal replacement by amyloid deposits, first compromising spermatogenesis in $100 \%$ of the patients and then endocrine function in $50 \%$.

In the literature it is known that the onset of hypergonadotropic hypogonadism can follow nonobstructive azoospermia in primary testicular failure. ${ }^{15}$ Tubular dysfunction may occur with normal testosterone production initially due to higher sensitivity to damage to seminiferous tubules rather than Leydig cells. ${ }^{18}$

In this series testicular volume was normal or high compared to that in other pathological conditions associated with impaired spermatogenesis that lead to micro-orchidism, including primary hypogonadism due to congenital abnormalities, Klinefelter's syndrome, myotonic dystrophy, cryptorchidism and abnormal karyotypes. These findings confirm previous case report data on the size of amyloidotic testicles. ${ }^{5-8,19}$ Briefly, testicular involvement appears to be an early and clinically relevant feature of apoA-I amyloidosis compared to other systemic forms of the disease.

In the literature histological data indicate a similar distribution of amyloid deposits in the testicular parenchyma apart from the protein precursor involved..$^{17}$ To our knowledge the reasons for the peculiar tissue targeting of apoA-I amyloid deposits are presently unknown. In particular no association with any previous testicular pathology was found. We hypothesize that the high concentration of the amyloid precursor in the testicular extracellular milieu might be due to the specific functional and metabolic properties of apoA-I. In fact, this protein has a key role in cholesterol trafficking, particularly in steroidogenic tissues. ${ }^{20}$ ApoA-I removes excess cholesterol from Sertoli's cells through the adenosine triphosphate-binding cassette transporter-A1 receptor and it supplies cholesterol to steroidogenic cells through the scavenger receptor class B type-I. It is possible that conformational changes occurring during the process of lipid exchange might contribute to the proteolytic remodeling of the protein when mutated, promoting the generation of $\mathrm{N}$-terminal peptides and triggering the fibrillogenetic process.

\section{CONCLUSIONS}

This study clearly points out that infertility with or without complete hypogonadism is an early and clinically relevant finding in hereditary Apo A-I amyloidosis, particularly in association with the Leu75Pro variant of this protein. Testicular involvement is associated with normal or high testicular volume and it may be the first or single manifestation of disease in young patients. Dramatic impairment of fertility may occur from the third decade of life. Progression of spermatogenic deterioration to complete azoospermia may happen in about 2 years. Thus, semen analysis should be advised annually in young, asymptomatic carriers of the mutation, whereas testicular biopsy is suggested at the onset of spermatogenic impairment to increase the chance of sperm retrieval. Moreover, evaluation of gonadic function should be done annually, also in older patients with known renal or hepatic damage, to start testosterone administration when necessary. In cases of infertility, nonobstructive azoospermia and macro-orchidism with complete or subclinical hypogonadism apoA-I amyloidosis must be suspected even in the absence of a significant family history.

\begin{tabular}{|c|c|}
\hline \multicolumn{2}{|r|}{ Abbreviations and Acronyms } \\
\hline $\mathrm{AA}$ & $=$ reactive amyloidosis \\
\hline $\mathrm{AL}$ & $=$ Ig light-chain amyloidosis \\
\hline Apo & $=$ apolipoprotein \\
\hline ED & $=$ erectile dysfunction \\
\hline $\mathrm{FSH}$ & $=$ follicle-stimulating hormone \\
\hline $\mathrm{fT}$ & $=$ free testosterone \\
\hline Gyn & $=$ gynecomastia \\
\hline Inf & $=$ infertility \\
\hline Leu75Pro & $=$ leucine 75 proline \\
\hline LH & $=$ luteinizing hormone \\
\hline rLib & $=$ decreased libido \\
\hline SHBG & $=$ sex hormone binding globulin \\
\hline $\mathrm{T}$ & $=$ total testosterone \\
\hline
\end{tabular}

\section{REFERENCES}

1. Merlini G and Bellotti V: Molecular mechanisms of amyloidosis. N Engl J Med 2003; 349: 583.

2. Kyle RA and Gertz MA: Primary systemic amyloidosis: clinical and laboratory features in 474 cases. Semin Hematol 1995; 32: 45.

3. Ben-Chetrit E and Levy M: Reproductive system in familial Mediterranean fever: an overview. Ann Rheum Dis 2003; 62: 916

4. Haimov-Kochman R, Prus D and Ben-Chetrit E: Azoospermia due to testicular amyloidosis in a patient with familial Mediterranean fever. Hum Reprod 2001; 16: 1218. 
5. Handelsman DJ, Yue DK and Turtle JR: Hypogonadism and massive testicular infiltration due to amyloidosis. J Urol 1983; 129: 610.

6. Bonacina R, Virgili G, Rosi P, Vespasiani G, Capodicasa E and Micali F: Testicular and cardiac amyloidosis. Scand J Urol Nephrol 1992; 26: 297.

7. Kanada DJ and Sharma OP: Long-term survival with diffuse interstitial pulmonary amyloidosis. Am J Med 1979; 67: 879.

8. Schrepferman CG, Lester DR and Sandlow JI: Testicular amyloid deposition as a cause of secondary azoospermia. Urology 2000 ; 55: $145 \mathrm{i}$.

9. Obici L, Bellotti V, Mangione P, Stoppini M, Arbustini E, Verga L et al: The new apolipoprotein A-I variant Leu $^{174} \rightarrow$ Ser causes hereditary cardiac amyloidosis, and the amyloid fibrils are constituted by the 93-residue $\mathrm{N}$-terminal polypeptide. Am J Pathol 1999; 155: 695.

10. Murphy CL, Wang S, Weaver K, Gertz MA, Weiss DT and Solomon A: Renal apolipoprotein A-I amyloidosis associated with a novel mutant Leu64Pro. Am J Kidney Dis 2004; 44: 1103.

11. Obici L, Palladini G, Giorgetti S, Bellotti V, Gregorini G, Arbustini $\mathrm{E}$ et al: Liver biopsy discloses a new apolipoprotein A-I hereditary amyloidosis in several unrelated Italian families. Gastroenterology 2004; 126: 1416.

12. Gregorini G, Izzi C, Obici L, Tardanico R, Röcken C, Viola BF et al: Renal apolipoprotein A-I amyloidosis: a rare and usually ignored cause of hereditary tubulo-interstitial nephritis. J Am Soc Nephrol 2005; 16: 3680.
13. Vermeulen A, Verdonck L and Kaufman JM: A critical evaluation of simple methods for the estimation of free testosterone in serum. J Clin Endocrinol Metab 1999; 84: 3666.

14. Schiff JD, Li PS and Goldstein M: Correlation of ultrasonographic and orchidometer measurements of testis volume in adults. BJU Int 2004; 93: 1015.

15. Griffin JE and Wilson JD: Disorders of the testes and the male reproductive tract. In: Williams Textbook of Endocrinology, 10th ed. Edited by PR Larsen, HM Kronenberg, S Melmed and KS Polonsky. Philadelphia: WB Saunders 2003; chapt 18, pp 709-770.

16. Coriu D, Dispenzieri A, Stevens FJ, Murphy CL, Shuching W, Wiess DT et al: Hepatic amyloidosis resulting from depositing of the apolipoprotein A-I variant Leu75Pro. Amyloid: J Protein Folding Disord 2003; 10: 215.

17. Özdemir BH, Özdemir OG, Özdemir FN and Özdemir AI: Value of testis biopsy in the diagnosis of systemic amyloidosis. Urology 2002; 59: 201.

18. Winters SJ: Male hypogonadism. In: Endocrinology and Metabolism. Edited by A Pinchera, X Bertagna, J Fischer, L Groop, J Schoemaker and M Serio. New York: McGrawHill 2001; chapt 40, pp 399-412.

19. Casella R, Nudell D, Cozzolino D, Wang H and Lipshultz LI: Primary testicular amyloidosis mimicking tumor in a cryptorchid testis. Urology 2002; 59: 445.

20. Marcel YL and Kiss RS: Structure-function relationships of apolipoprotein A-I: a flexible protein with dynamic lipid associations. Curr Opin Lipidol 2003; 14: 151. 